\title{
Comparison of Multi-View Face Recognition using DCT and Hybrid DWT of Score Fusion under Uncontrolled Illumination Variation
}

\author{
Manisha J Kasar \\ M.Tech Student (CE) \\ Computer Department, MPSTME \\ NMIMS, Shirpur, Dist :Dhule, Maharashtra, \\ India
}

\author{
Nitin S.Choubey \\ P.hd (Computer) \\ Computer Department, MPSTME \\ NMIMS, Shirpur, Dist :Dhule, Maharashtra, \\ India
}

\begin{abstract}
A facial recognition system is one of the biometric applications for automatically identifying or verifying a person from a digital image or a video frame from a video source. Now a day's face recognition system is recognize the face using multiple-views of faces, for detecting each view of face such as left, right, front, top, and bottom. The proposed face recognition system consists of a novel illuminationinsensitive preprocessing method, a hybrid discrete Wavelet Transform (HDWT) for feature extraction and Density based Score Fusion Technique for matching. First, in the preprocessing stage different stages are followed like Gamma Correction, DOG(Difference of Gaussion)filtering and contrast equalization which transforms, a face image into an illumination-insensitive image, Then, for feature extraction of complementary classifiers, multiple face models based upon HDWT are applied. To do the feature extraction this system uses DCT Wavelet transform to generate the feature vectors of the query and database images. Euclidean distance is used as similarity measure to compare the image. Finally, to combine scores from multiple complementary classifiers, a log likelihood ratio-based score fusion scheme is applied. The proposed system uses the ORL face database, and compare with DCT method. Using ORL database the Hybrid wavelet has achieves smaller size feature vectors which is performing better with $98.55 \%$ accuracy and less computation time require for recognizing image which is $19.0645 \mathrm{msec}$ to $24.7653 \mathrm{msec}$.Which is less as compare to DCT method, DCT method achieves $96.45 \%$ accuracy and computational time $26.2349 \mathrm{msec}$ to $33.0234 \mathrm{msec}$ which is more as compare to proposed method..
\end{abstract}

\section{Keywords}

Face Recognition, Preprocessing, Feature extraction, Score Fusion, Biometrics, Multi-view face recognition, DCT, DCT Wavelet, Euclidean distance

\section{INTRODUCTION}

Image processing is widely used in many applications, including medical imaging, industrial manufacturing, and security systems, face recognition, human recognition, figure print recognition in cyber crime, military application, and medical diagnosis. Now days we need to maintain global security Information, in every organization or individual wants to improve their existing security system. Most of the people need better security system which gives complete security solution. Recently, technology became available to allow verification of true individual identity. This technology is based in a field called "biometrics". Biometrics is a technique for identifying people by using a unique physiological characteristic, such as a fingerprint, eye, face, etc. or behavioral characteristics, e.g., voice and signature etc. Biometrics is the use of computers to recognize people. Among the various biometric ID methods, the physiological methods such as fingerprint, face, DNA are more stable than methods in behavioral category like keystroke, voice print etc.. Face recognition is one of the biometric methods that to have the merits of both high accuracy and low intrusiveness. For this reason, the face recognition has drawn the attention of researchers in fields from security, Psychology, and image processing, to computer vision. Many algorithms have been proposed for face recognition, Face recognition has also proven useful in other multimedia information processing areas. Facial recognition analyzes the characteristics of a person's face images input through a digital video camera or online face capturing.

In this paper Hybrid Discrete Wavelet Transform (HDWT) is used for extracting feature from face image and DCT (Discrete Cosine Transform) is used for comparison with proposed method. The logic behind that DCT is a good approximation of principal component extraction, which helps to process and highlight the signal frequency features. The Discrete-Cosine Transform (DCT) has found popularity due to its comparative concentration of information in a large number of coefficients, and increased tolerance to variation of illumination. The most relevant coefficients for recognition are selected using the technique of low frequencies selection. To classify a face, its DCT coefficients are extracted. Next, the distances between the DCT coefficients of the face to be classified and the DCT coefficients of all faces in the database are calculated. The shortest of all distances will probably be associated with faces of the same person; therefore the face under classification will be classified as belonging to the person whose face in the database has the shortest distance to the face to be classified [20].

In this paper, the section II gives Structure and Procedure of Face Recognition System, section III gives Literature Survey of Face Recognition Techniques and section IV gives comparative method section $\mathrm{V}$ gives the proposed work for system. The rest of the paper Describes illumination insensitive representation as a preprocessing method is discussed in Section VI, In Sections VII Feature Extraction using hybrid Discrete Wavelet Transform from multiple face models is explained, In Section VIII, log-likelihood-ratebased Density based score fusion is presented, in section IX Experimental results and discussion is discussed and the 
Section X concludes the proposed work and comparative method.

\section{STRUCTURE AND PROCEDURE OF FACE RECOGNITION SYSTEM}

The face recognition procedure generally separates into three steps: Face Detection, Feature Extraction, and Face Recognition [1].

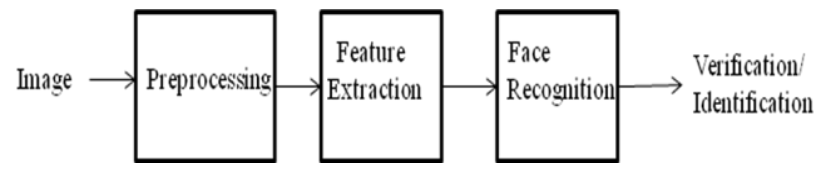

Fig 1 Architecture of face recognition system [1]

As shown in fig 1 the first task of the face recognition system is capturing image by video or by camera and this image is given to the further step of face recognition system that is:

\section{Face Detection:}

The main function of this step is to determine whether the human faces appear in given image or not and where the face is located at. The outputs of this step are patches containing each face in the input image, face alignment are performed to justify the scales and orientations of these patches. These step is working as the preprocessing for face recognition, face detection could be used for region of interest detection, retargeting, video and image classification, etc.

\section{Feature Extraction:}

In this step feature extractions are performed to do information packing, dimension reduction, salience extraction, and noise cleaning. After this step, a face patch is usually transformed into a vector with fixed dimension or a set of fiducial points and their corresponding locations.

\section{Face Recognition:}

This step analyzes the representation of each face; this last step is used to recognize the identities of these faces. In order to achieve automatic recognition, a face database is required to build. For each person, several images are taken and their features are extracted and stored in the database. Then when an input face image comes in, then perform face detection and feature extraction, and compare its feature to each face class stored in the database. There have been many researches and algorithms proposed to deal with this classification problem. There are two general applications of face recognition, one is called identification and another one is called verification. Face identification means given a face image, can be used to determine a person's identity even without his knowledge or consent. While in face verification, given a face image and a guess of the identification, the system must to tell about the true or false about the guess.

\section{SURVEY OF FACE RECOGNITION TECHNIQUES}

Now a day's face recognition system is recognize the face using multiple-views of faces, these Multi-view face recognition techniques has proposed by some authors for detecting each view of face such as left, right, front, top, and bottom using some methods which is discussed below

Several methods are describe in literature are also based on mathematical transform such as DCT and wavelet transform. The method proposed by Ramamoorthi and Pat Hanrahan[2] and Amnon Shashua and Tammy Riklin-Raviv[3] color based; image based re-rendering recognition which is proposed by Ravi. This method is has problem of pose variation and that is solve by Hybrid PCA method, But this method does not work well when large number of database is used for face recognition. Hybrid-PCA method is suggested by M. Savvides, B. Kumar, and P. Khosla[5], which works better when large database is consider for face recognition, this method is also work on partial faces, as well as illumination condition but the accuracy of recognizing faces is somewhat poor. This accuracy problem is slightly resolved in Kernel based face recognition method which has been suggested by Xiaoyang Tan and Bill Triggs[8],these method have the problem of additional feature set which is resolve in likelihood ratio based score fusion technique which is proposed by Karthik Nandakumar[19], which increase the recognition rate without need of additional parameters. Some of these methods works only on local features of face image, but in the Fisher Linear Discremenant method which is suggested by $\mathrm{Yu} \mathrm{Su}$, Shiguang Shan, X. Chen, and W. Gao[12], it works on both the features like local and global features of face image, but the problem of illumination condition is not solve using this method. This problem is solve by Hybrid Fourier feature method suggested by Wonjun Hwang, Haitao Wang, Hyunwoo Kim[13], This method works well on different illumination condition, works only in frequency domain. Because of this reason the computation time is increase for extracting the features from face. And this also effects on efficient face recognition performance. The DCT method has been used for to do comparison with proposed method which is in feature extraction

\section{DCT (DISCRETE COSINE TRANSFORM)}

The Discrete Cosine Transform (DCT) is an invertible linear transform that can express a finite sequence of data points in terms of a sum of cosine functions oscillating at different frequencies. The original signal is converted to the frequency domain by applying the direct DCT transform and it is possible to convert back the transformed signal to the original domain by applying the inverse DCT transform. After that the original signal has been transformed into DCT coefficients which reflect the importance of the frequencies that are present in it. The first coefficient referred as signal's lowest frequency, known as the DC-coefficient, and usually carries the majority of the relevant information from the original signal. The last coefficient is referred as a signal's higher frequencies [20]. These higher frequencies generally represent more detailed or fine information of signal and probably have been caused by noise. The rest of the coefficients carry different information levels of the original signal. Discrete cosine transform is made up of cosine functions taken over half the interval and dividing this interval into $\mathrm{N}$ equal parts and sampling each function at the center of these parts, the DCT matrix is formed by arranging these sequences row wise. DCT transform is used to generate the feature vectors. The DCT definition of 2D sequence of Length $\mathrm{N}$ is given in equation (1) using which the DCT matrix is generated [18]. For the DCT, any image block $A_{m, n}$, of size $M x N$, can be written as the sum of the MN functions of the form [26]:

$$
\alpha_{p} \alpha_{q} \cos \left(\frac{\pi(2 m+1) p}{2 M}\right) \cos \left(\frac{\pi(2 n+1) q}{2 N}\right)
$$

For $0 \leq \mathrm{pM}-1,0 \leq \mathrm{N}-1$

These are the basis functions of the DCT [26].

$B_{p \cdot q}=\sum_{m=0}^{M-1} \sum_{n=0}^{n N-1} A_{m n} \alpha(p) \alpha(q) \cos \left[\frac{(2 m+1) p \pi}{2 M}\right] \cos \left[\frac{(2 n+1) q \pi}{2 N}\right]$ 
Where,

$$
\begin{aligned}
& \alpha(p)=\left\{\begin{array}{l}
\sqrt{\frac{1}{N}}, \text { for } p=0 \\
\sqrt{\frac{2}{N}}, \text { for } p=1,2, \ldots M-1
\end{array}\right. \\
& \alpha(q)=\left\{\begin{array}{l}
\sqrt{\frac{1}{N}} \text {, for } q=0 \ldots(3) \\
\sqrt{\frac{2}{N}, \text { for } q=1,2, \ldots N-1}
\end{array}\right.
\end{aligned}
$$

And $\mathrm{A}_{\mathrm{mn}}$ is the $\mathrm{MxN}$ input matrix. The result is MN 2-D DCT coefficients. DCT method uses a selection of the 2dimensional DCT coefficients from each block to construct the feature vector for an image. For the best identification rates, the coefficients selected should be the most significant in representing important and distinguishing image details. Using one coefficient to represent each block, all images of the database were represented as feature vectors, and then the recognition rates were determined. Here feature value is calculated using DCT transform for training image which have image size is $256 \times 256$.

\begin{tabular}{|r|r|r|r|}
\hline & \multicolumn{1}{|c|}{1} & \multicolumn{1}{c|}{2} & \multicolumn{1}{c|}{3} \\
\hline 1 & $1.4616 \mathrm{e}+03$ & $1.6327 \mathrm{e}+03$ & $2.2652 \epsilon$ \\
\cline { 1 - 1 } 2 & -4.5628 & -77.7391 & $-144 . \varepsilon$ \\
\cline { 1 - 3 } & 847.6761 & 853.1341 & 311.1 \\
\cline { 1 - 1 } 4 & 222.3156 & 219.5367 & $103 . \varepsilon$ \\
\cline { 1 - 1 } 5 & -908.7193 & -804.7309 & $-178 . \varepsilon-$ \\
\hline
\end{tabular}

\section{Fig 4. The feature vector of image using DCT Transform}

Each block is a subset of the image, with the number of identity distinguishing details contained in each block, related to the fraction of the image it includes. Each block tells about the feature vector of each pixel of image, which is prepared for all the database images. Once the feature databases are prepared system is tested with query image. Feature extraction of query image will be done in same manner as it does for the database images. Here ORL database is used to test the image features. Similarity measure Euclidean distance is applied to compare the query image with the database images for similarity. Retrieval results are based on the criterion of sorting the Euclidean distances [20]. But to calculate the feature vector for whole image requires more time, which is difficult to maintain that large data for all database image and also the performance measurement of DCT method is poor. To remove these limitations the proposed method is discussed below which helps us to increase the performance of face recognition system and reduced computational time to recognize face image.

\section{PROPOSED WORK}

The above discussed methods have some limitations which have solved by many researchers, but there are still some other issues in face recognition like computational time and efficiency of recognizing the faces from large databases. Automatic face recognition is an important vision task with many practical applications such as biometrics, video surveillance, image retrieval, and human computer interaction. One major issue for face recognition is how to ensure recognition accuracy for a large data set captured in various conditions. In this paper Olivetti Research Ltd (ORL) database of faces is used to test the performance of system. One is taken in a controlled studio setting while the other is captured in uncontrolled illumination conditions such as hallways, atria, or outdoors. To overcome the problem of uncontrolled environmental problem using preprocessing method which is discussed in section V, a hybrid DWT (Discrete Wavelet Transform) will be used for feature extraction which reduces the computational complexity, because work in both frequency and time domain, DWTs also have higher flexibility, better compression ratio and performance, This approach combines multiple classifiers with complementary features instead of improving the accuracy of a single classifier. Illumination insensitive preprocessing and density based score fusion technique is combine into the proposed face recognition system which will give efficient face recognition performance. The following figure shows the block diagram of the proposed system.

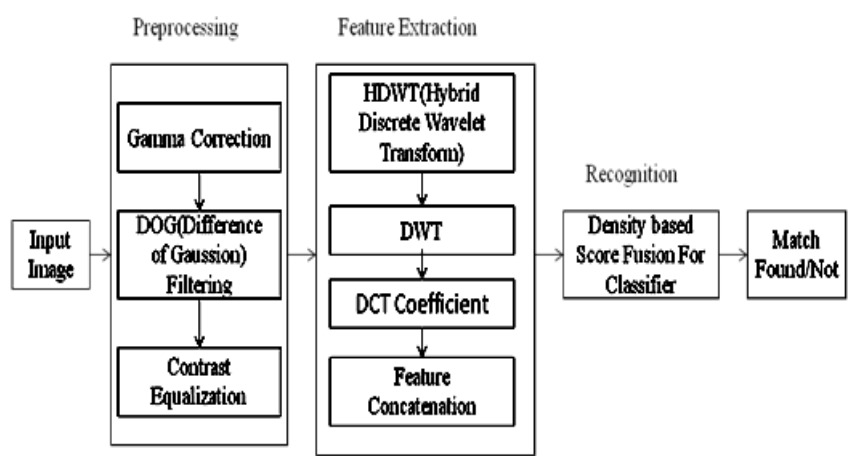

Fig 5. Block Diagram of Proposed System

Above block diagram shows the structure of proposed system which includes three steps: preprocessing, feature extraction and recognition. First step of proposed multiple face model recognition is preprocessing which includes Gamma Correction, Difference of Gaussion(DOG) and Contrast Equalization. The preprocessing method is used here to solve one of the common problems in face images, due to a real capture system i.e. lighting variations. The different stages include gamma correction, Difference of Gaussian (DOG) filtering and contrast equalization. Gamma correction enhances the local dynamic range of the image in dark or shadowed regions while compressing it in bright regions and is determined by the value of $\gamma$. DOG filtering is a grey scale image enhancement algorithm that eliminates the shadowing effects. Contrast equalization rescales the image intensities to standardize a robust measure of overall intensity variations [16]. In feature Extraction Hybrid Discrete Wavelet transform is used which is used for person classification and any invariance in the face images against environmental changes. In recognition step as we having the set of complementary classifiers, then build a unified classifier combining these complementary classifiers. The purpose of this classifier is to construct a strong classifier by suitably combining a set of classifiers. The information each classifier extracts is well summarized in the score each classifier produces. [9].

\section{PREPROCESSING METHOD UNDER VRYING ILLUMINATION CONDITION}

This preprocessing method combines the features of gamma correction, DOG filtering and contrast equalization 
techniques. Over all stages of proposed preprocessing method is shown in Fig.3 [20].

Input $\rightarrow \begin{gathered}\text { Gamma } \\ \text { Correction }\end{gathered} \rightarrow \begin{aligned} & \text { DOG(Difference of } \\ & \text { Gaussion) Filtering }\end{aligned} \rightarrow \rightarrow \begin{gathered}\text { Contrast } \\ \text { Equalization }\end{gathered} \rightarrow$ Output

Fig 6.The Stages of Preprocessing method [16]

\subsection{Gamma Correction}

Gamma Correction is used to display an image accurately on computer screen. It controls overall brightness and darkness of image. Image which is not properly corrected can look either light or too dark. And also trying to reproduce colors accurately which using gamma. Gamma correction changes not only the brightness, but also the ratios of red to green to blue. If gamma correction is done properly for the computer system, then the output should accurately reflect in image input. Gamma Correction is a nonlinear gray-level transformation that replaces gray-level I with the gray level $I^{1 /} / Y$ and is given by,

$$
I=I^{1 / y}
$$

The gamma value is ranges from $0-1$. This enhances the local dynamic range of the image in dark or shadowed regions while compressing it in bright regions [20].

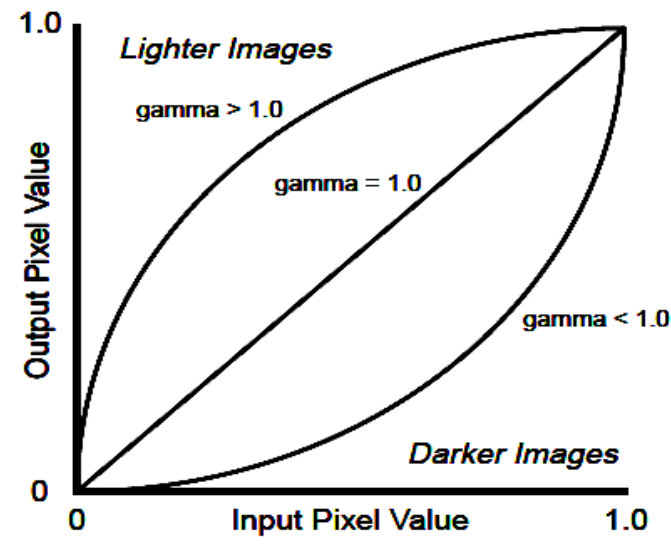

Fig 7 Gamma Curve [20].

This curve tells about the pure black parts of the image as black and the white parts as white, while adjusting the values in between in a smooth manner. The overall structure of an image can be lighter or darkener depending on the gamma value used, while maintaining the dynamic range of the image. In Figure 5.a, the pixel values range from 0.0 represents pure black, to 1.0, which represents pure white. As the figure shows, if gamma values of less than 1.0 image is represented as darker an image. And if Gamma values greater than 1.0 image is represented as lighter image and if a gamma value equal to 1.0 produces no effect on an image. Here Gamma $=0.4$ is used as the default setting [20]. As here I m using gamma value is 0.4 , if gamma value is less than 0.4 image is darker image and if gamma is greater than 0.4 images is lighter image, and when gamma value equal to 0.4 the image will display as smoother image.

\subsection{Difference of Gaussian (DOG) Filtering}

As Gamma correction does not remove the overall intensity gradients such as shading effects. For that reason Difference of Gaussians is used. It is a feature enhancement algorithm that involves the subtraction of one blurred version of an original image from another, less blurred version of the original image. In the simple grayscale images, the blurred images are obtained by convolving the original grayscale images with Gaussian kernels having differing standard deviations. Blurring an image using a Gaussian kernel has only the high frequency spatial details. Subtracting one image from the other preserves spatial information that lies between the ranges of frequencies that are preserved in the two blurred images. Thus, the difference of Gaussians is a band-pass filter that discards all but a handful of spatial frequencies that are present in the original grayscale image. The Difference of Gaussian (DOG) can be used to increase the visibility of edges and other detail present in image. The Difference of Gaussians algorithm removes high frequency detail that often includes random noise and this approach could be found well suitable for processing images with a high degree of noise [21].

The DOG Filter is defined as follows:

$$
\operatorname{DOG}(x, y)=\frac{1}{2 \pi \sigma_{1}^{2}} e^{\frac{x^{2}+y^{2}}{2 \sigma_{12}}}-\frac{1}{2 \pi \sigma_{2}^{2}} e^{\frac{x^{2}+y^{2}}{2 \sigma_{2}^{2}}} \ldots
$$

The values of $\sigma_{1}$ and $\sigma_{2}$ are chosen as 1.0 and 2.0 respectively for this system. This effect leads to the reduction in the overall contrast produced by the operation and hence the contrast has to be enhanced in the subsequent stages.

\subsection{Contrast Equalization}

This is final stage of the preprocessing method which rescales the image intensities. Contrast equalization is contrast enhancement technique which increases the global contrast of many images. This allows for areas of lower contrast to gain a higher contrast. The method is useful in images with backgrounds and foregrounds that are both bright or both dark. This stage includes Top-hat and Bottom-hat filtering can be used together to enhance contrast in an image. Top-hat filtering can be used to uneven illumination when the background is dark. It is used to extracting the light object. Bottom hat is used to extract dark object from light background. The procedure is to add the original image to the Top-Hat filtering image and then subtract the bottom-hat filtered image.

$$
\begin{gathered}
T_{\text {hat }}=X-(X \circ K) \\
B_{\text {hat }}=(X \bullet K)-X
\end{gathered}
$$

where $\mathrm{X}$ is an image and $\mathrm{K}$ is a structuring element ,structuring element in a is a shape, used to probe or interact with a given image, with the purpose of drawing conclusions on how this shape fits or misses the shapes in the image. The figure 5.1 shows the structure of preprocess stages after applying on image.

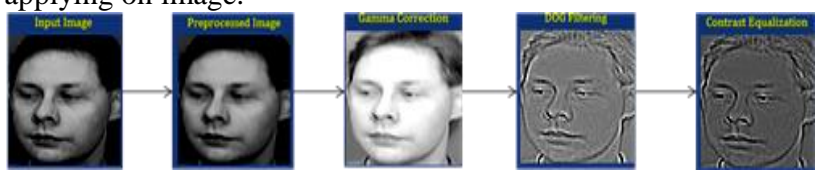

Fig 8 Structure of preprocess image

The above diagram represents the overall structure of input image after performing all preprocesses stages. First, it will select an image form database then perform the smoothing filter operation on image, then it generates the smooth image but this smooth image cannot reduce the dark and light part of image accurately for that reason next we will apply Gamma Correction step on it. After generating result for gamma correction it will further apply Difference of Gaussion (DOG) 
Filter which reduces the all showing effect from image and it will increase the visibility of edges and other details present in image, but using this stage it will generate the contrast in mage and hence the contrast has to be enhance in contrast equalization stage. This stage increases the global contrast of image. The method is useful in images with backgrounds and foregrounds that are both bright or both dark which is shown in Contrast equalization image, Further this image will given as a input for performing feature extraction operation that describes in below section.

\section{HYBRID DISCRETE WAVELET TRANSFORM FOR FEATURE EXTRACTION}

Wavelets became popular in few past years in mathematics and digital signal processing area because of their ability to effectively represent and analyse data. There are many generalisations of original orthogonal wavelet systems. In addition to construct more general wavelet system, The ways how to construct discrete hybrid wavelet transforms (HWT), i.e. generalisation of discrete wavelet transforms (DWT) where basis is created by mixing 2 or more bases of existing DWT. Such approach is call hybridisation and to the resulting transform we refer as discrete hybrid wavelet transforms (HWT) [15].

In this paper to do the feature extraction this system uses DCT Wavelet transform to generate the feature vectors of the query and database images. DCT wavelet is applied over row mean vector of each block separately and 4 sets of DCT wavelet coefficients are obtained respectively [18]. Out of these few coefficients are selected from each block and arranged in consecutive order to form the feature vector of the image. Variable size feature vectors are formed by changing the number of coefficients selected from each row vector. These two different feature databases obtained using DCT wavelet is then tested using different query images. Euclidean distance is used for similarity measurement to compare the image features. Euclidean distance calculated is sorted into ascending order to count the images which are relevant to the query image. First separate the image into Approximate, Horizontal, vertical and diagonal planes and then decomposing the image plane into 4 blocks and applying DCT transform over row mean vectors of each block of it to obtain the information of the image. Same process is repeated with DCT wavelet transform over row mean vectors of each block of each plane. Discrete cosine transform is made up of cosine functions taken over half the interval and dividing this interval into $\mathrm{N}$ equal parts and sampling each function at the center of these parts, the DCT matrix is formed by arranging these sequences row wise [18]. This paper uses DCT transform to generate the feature vectors. Wavelets are mathematical functions that cut up the data or signal into different frequency components by providing a way to do a time frequency analysis. This paper has proposed a new algorithm to represent the feature vectors in the form of discrete cosine wavelet transform coefficients for image database. The DCT definition of 2D sequence of Length $\mathrm{N}$ is given in equation (7) using which the DCT matrix is generated [18]. The generalized algorithm which can generate wavelet transform of size $\mathrm{N}^{2} \mathrm{xN}^{2}$ from any orthogonal transform of size $\mathrm{NxN}$ is applied to DCT matrix and DCT Wavelet is developed which satisfies the condition of orthogonal transforms given in equation (9) [18]. Once the Discrete Cosine Transform Wavelet is generated following steps are followed to form the feature vectors of the images.

$$
F[k, l]=\sum_{m=0}^{M-1} \sum_{n=0}^{n N-1} f[m, n] \alpha(k) \alpha(l) \cos \left[\frac{(2 m+1) k \pi}{2 M}\right] \cos \left[\frac{(2 n+1) l \pi}{2 N}\right]
$$

$\alpha(k)=\left\{\begin{array}{l}\sqrt{\frac{1}{N}, \text { for } k=0} \\ \sqrt{\frac{2}{N}, \text { for } k=1,2, \ldots N-1} \ldots \ldots(8)\end{array}\right.$

Orthogonal: DCT Wavelet transform is said to be orthogonal if the following condition is satisfied [19].

$[\mathrm{DCTW}][\mathrm{DCTW}]^{\mathrm{T}}=[\mathrm{D}] \ldots \ldots \ldots \ldots(9)$

\subsection{ALGORITHM for DCT WAVELET [19]:}

1. Separate image into approximate, Horizontal, and vertical plane.

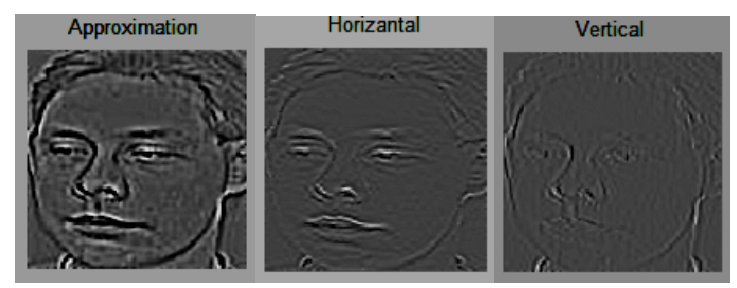

2 Divide each plane of image into four blocks A1, A2, A3 and A4 of all equal sizes.

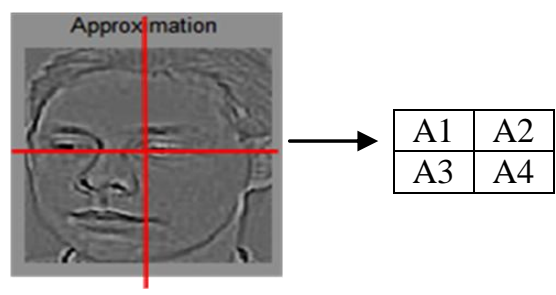

3 For each block calculate the row mean vectors.

\begin{tabular}{|l|l|l|c|c|}
\hline 145 & 158 & $\ldots \ldots$ & 165 & $(145+158+\ldots .+165) / n$ \\
\hline 122 & & & $\longrightarrow$ & \\
\hline 130 & & & $\longrightarrow$ & \\
\hline 178 & 220 & $\ldots \ldots$ & 160 & $\rightarrow$ \\
\hline
\end{tabular}

4. Applying a DCT wavelet over all row mean vectors of all four blocks of each plane of all database images and new DCT Wavelet feature database is prepared.

5. Representation of feature vectors is explained as follows: Select a few DCT wavelet coefficients from each row vector of all four blocks of each plane and arrange them in single vector in consecutive order. It gives the feature vector of that particular plane. Similar procedure is followed to get the feature vector for all three planes Approximate, Horizontal, Vertical and diagonal. Feature vectors for planes Approximate, Horizontal, Vertical and diagonal plane are obtained using above procedure and two feature vector databases are created for all the database images using DCT wavelet. 
6. Once the feature databases are prepared system is tested with query image. Feature extraction of query image will be done in same manner as it does for the database images.

Similarity measure Euclidean distance given in equation (10) is applied to compare the query image with the database images for similarity [19].

$$
D_{Q I}=\sqrt{\sum_{i=1}^{n}\left(F Q_{i}-F I_{i}\right)^{2}}
$$

7. Retrieval results are based on the criterion of sorting the Euclidean distances in ascending order and selecting images with respect to minimum distances from nearest distances sorted in ascending order for all database images [19].

So that applies DWT for the DCT co-efficient. The image it's converted as signal while extracting features.

\section{RECOGNITION USING DESITY BASED SCORE FUSION}

Once we have a set of complementary classifiers, then we build a unified classifier combining these complementary classifiers. To keep as much information each classifier extracts as possible, and at the same time the combination should be easy to implement. The information of each classifier extracts is well summarized in the score each classifier produces. Hence, combining the classifiers can be achieved by processing the set of scores produced by component classifiers and generating a new single score value. This process is call as "score fusion." A multiple face model that consists of different face models with different eye distances in the same image size. Here the different face models with the same image sizes, $256 \times 256$, are constructed with different eye distances and the Gamma Correction, DOG (Difference of Gaussion) Filtering, Contrast Equalization these stages are applied to each normalized face image. Then we have Approximate, Horizontal, and Vertical face models after applying HDWT on preprocess image. The Approximate face model is formed to analyze the internal components of a face, such as the eyes, nose, and mouth, while the Horizontal face model includes the general structures of a face and the external components such as hair, ear, and jaw-line. The last one, the Vertical face model, is a compromise between the approximate model and the Horizontal model. Each face model can play an inherent role for the others in the face recognition system. For example, the approximate face model is robust to background and hair style changes but sensitive to pose changes. On the other hand, the Horizontal face model shows the opposite tendency. In the end, we can have different classifiers, and each similarity score is calculated by a normalized correlation. The equation of the normalized score between two features $Y_{\mathrm{i}}^{a}$ and $Y_{\mathrm{i}}^{\mathrm{b}}$ in the $\mathrm{i}^{\text {th }}$ classifier is defined as [13].

$$
S\left(Y_{i}^{a}, Y_{i}^{b}\right)=\frac{\sum_{k=1}^{K} Y_{i k}^{a} Y_{i k}^{b}}{\left\|Y_{i}^{a}\right\| \cdot\left\|Y_{i}^{b}\right\|}
$$

Here in this paper we considering the score fusion method based on weighted sum method, this method is based on Equal Error Rate (EER). The EER-based score fusion computes a weighted sum of scores, where the weight is a measure of the discriminating power of the component classifier. To combine the scores is to compute a weighted sum as follows [13]:

$$
S=\sum s_{i} w_{i}
$$

Where the weight $\mathrm{w}_{\mathrm{i}}$ is the amount of confidence we have in the $\mathrm{i}^{\text {th }}$ classifier and its score $\mathrm{s}_{\mathrm{i}}$. One drawback of the weighed sum method is that the scores generated by component classifiers may have different physical or statistical meaning and different ranges. Hence, we should make sure that the range of each score is normalized appropriately [13]. The LLR-based fusion method is more principled than the weighted sum method in that the LLR-based fusion method is derived from the optimal likelihood ratio test. The method's decision boundary is nonlinear, there by being able to perform more complex classification. Suppose we have a set of scores $\mathrm{s}_{1} \ldots \ldots . \mathrm{s}_{\mathrm{n}}$ computed by $\mathrm{n}$ classifiers. Now the problem is to decide whether the query-target pair is from the same person or not based upon these scores. We can cast this problem as the following [13]:

$$
\begin{array}{r}
P\left(s_{1}, \ldots \ldots \ldots, s_{n} \mid \text { diff }\right) \\
P\left(s_{1}, \ldots \ldots \ldots, s_{n} \mid \text { same }\right) \ldots \ldots .
\end{array}
$$

Where $P\left(s_{1}, \ldots \ldots \ldots, s_{n} \mid\right.$ diff $)$ is the distribution of the scores when the query and target are from different persons, and $P\left(s_{1}, \ldots \ldots, s_{n} \mid\right.$ same $)$ same is the distribution of the scores when the query and target are from the same person. If we know the two densities $P\left(s_{1}, \ldots \ldots \ldots, s_{n} \mid\right.$ diff $)$ and $P\left(s_{1}, \ldots \ldots \ldots, s_{n} \mid\right.$ same $)$ the log-likelihood ratio test achieves the highest verification rate for a given false accept rate. However, the true densities $P\left(s_{1}, \ldots \ldots, s_{n} \mid\right.$ diff $)$ and $P\left(s_{1}, \ldots \ldots, s_{n} \mid\right.$ same $)$ ar e unknown, so we need to estimate these densities observing scores computed from query-target pairs in the training data. Then, we model the $m_{\text {diff } f_{i}^{i}}$ distribution of $\mathrm{s}_{\mathrm{i}}$ as a Gaussian random variable with mean and variance $\sigma^{2}$ diff,$i_{i}$, and model $\left\{S_{i}\right\}_{i=1}^{n}$ as independent Gaussian random variables with density $P\left(s_{1}, \ldots \ldots \ldots, s_{n} \mid\right.$ diff $)=\pi N\left(s_{i} ; m_{\text {diff } i} ; \sigma_{\text {diff } i}^{2}\right)$. . (14)

Where,

$$
N\left(s_{i} ; m ; \sigma^{2}\right)=\left(1 / \sqrt{2 \pi \sigma^{2}}\right) \exp \left\{-\left((x-m)^{2} /\right.\right.
$$

$\left.\left.2 \sigma^{2}\right)\right\}$

the Gaussian density function. The parameters $m_{\text {diff } i i} \sigma_{\text {diff } i \text { i }}^{2}$ are estimated from the scores of the $\mathrm{i}^{\text {th }}$ classifier corresponding to non-match query-target pairs in the training database. Similarly, approximate density of $\left\{S_{i}\right\}_{i=1}^{n}$ by $\pi N\left(s_{i}, m_{\text {diff } i i} ; \sigma_{\text {diffii }}^{2}\right)$ and the parameters $\left(m_{\text {diff } i}, \sigma^{2}\right.$ diff $\left.i_{i}\right)$ and $\left(m_{\text {same } i j}, \sigma_{\text {same } i}^{2}\right)$ are computed from the scores of the $i^{\text {th }}$ classifier corresponding to match query-target pairs in the training database. The score fusion in (26.4955) depends upon the parameters that we estimate from the training data. Thus, this method is more sensitive to compare between the statistics of the training data and the test data than the weighted sum method. For instance, 
the score fusion in (26.4955) is affected by the shift of the

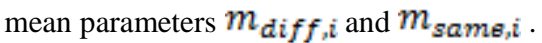

\section{EXPERIMENTAL RESULTS AND COMPARISION OF VARIOUS METHODS}

The results generated after implementing above proposed method. For generation of DCT and HDWT we made use of Cosine Transform and Wavelet transform. The results are shown below. The methods are tested with ORL database and compare with DCT method. Olivetti Research Ltd (ORL) database of faces. The database consists of 400 images, 10 views of each 40 person. There are 4 female and 36 male persons. Here we are considering the size of image is $256 \times 256$. Five images of each person were used for training and Five for testing, giving a total of 200 training and 200 test images. The comparative studies of above discussed methods are summarizing here. Here we are extracting the feature vectors of above discussed procedure based on DCT and HDWT. Once the Query is entered it is processed which explained above to extract its contents to form the feature vector. The Hybrid wavelet method first converts image into three planes Approximate, Horizontal and Vertical. This information is handled separately to form the feature vectors. As each plane is divided into 4 blocks and DCT transforms are applied to row mean vectors of each block which tells that the appearance of the image is taken into consideration while forming the feature vectors. By changing the size of the feature vectors using different sets of images computational time complexity is analyzed and it can be defined that computational time can be saved with smaller size feature vectors which are performing better as compared to the larger ones. But in DCT method it directly converts image in feature vector matrix which has large size feature vectors. To calculate this feature vector using DCT method requires more time to convert whole image in matrix and for that reason the performance of system is poor as compare to proposed method. This matrix is then used for similarity measurement using Euclidean distance; it will compare the query image with database images for the exact match. Euclidean distance is calculated for all of them, As DCT and Hybrid wavelet has been studied and compared with ORL database. The Hybrid wavelet has smaller size feature vectors which are performing better with $98.55 \%$ accuracy and less computation time require recognizing image which is $19.0645 \mathrm{msec}$ to 24.7653 msec. with comparison of DCT method. DCT method achieves $96.45 \%$ accuracy and computational time 26.2349 msec to $33.0234 \mathrm{msec}$ which is more as compare to proposed method. The performance graph for proposed method and DCT method is shown in below figure.

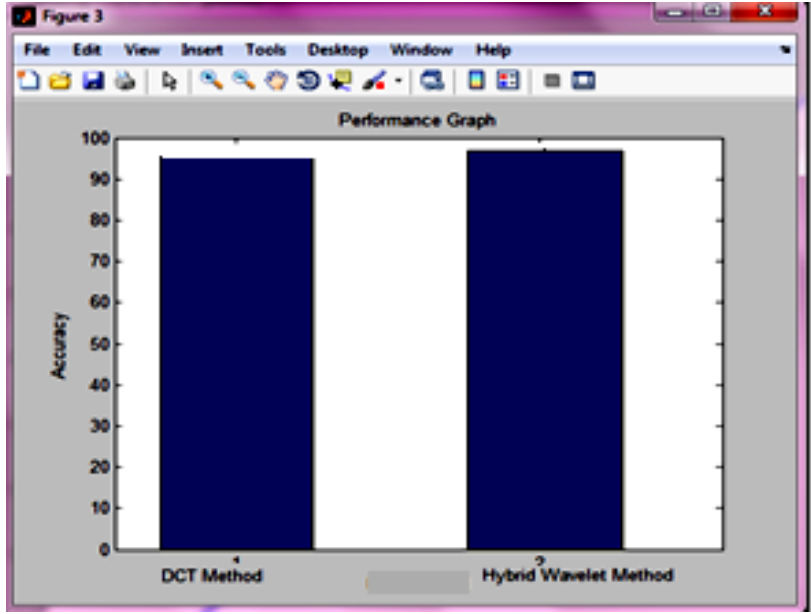

Fig 9 Performance analysis of DCT and HDWT method TABLE I: Compression of Accuracy and computational time measurement of DCT and Proposed Method with ORL database

\begin{tabular}{|l|l|l|}
\hline Name of Method & Accuracy & Computation time Per image \\
\hline DCT & $96.45 \%$ & $19.0645 \mathrm{msec}$ to $24.7653 \mathrm{msec}$ \\
\hline Proposed Method & $98.55 \%$ & $26.2349 \mathrm{msec}$ to $33.0234 \mathrm{msec}$ \\
\hline
\end{tabular}

\section{CONCLUSION}

The Multi Model face recognition system based on HDWT (Hybrid Discrete Wavelet Transform) and Density based Score fusion technique and DCT transform has been discussed in this paper. It mainly focuses on application and Score Fusion technique under uncontrolled illumination, and their performance analysis and comparative study. Both the methods are tested with ORL database. With the help of DCT method size of feature vector is more. By changing the size of feature vectors using different method computational time complexity is analyzed and it can be defined that computational time can be saved with smaller size feature vectors which are performing better as compared to the larger ones. Finally when we compare DCT and HDWT it can be noticed that HDWT is performing better. The proposed system achieves successful accuracy in face recognition under uncontrolled illumination situations. There is scope for further improvement so that these approaches can be used for variable image sizes, color, texture shape feature and overall average precision.

\section{ACKNOWLEDGMENTS}

This paper is part of the project implementation and results comparison for identified problem. Author thanks to Dr. M. V. Deshpande, Associate Dean, Mukesh Patel School of Technology Management \& Engineering, NMIMS, Shirpur, for his valuable guidance in performing this project work.

\section{REFERENCES}

[1] Wei-Lun Chao, "Face Recognition", GICE, National Taiwan University, March 2007.

[2] Ravi Ramamoorthi and Pat Hanrahan," On the relationship between radiance and irradiance: Determining the illumination from images of a convex Lambertian 
object", J. Opt. Soc. Amer., vol. 18, no. 10, pp. 24482459,2001 .

[3] Amnon Shashua and Tammy Riklin-Raviv," The quotient image: Class-based re-rendering and recognition with varying illuminations", IEEE Trans. Pattern Anal. Mach. Intell., vol. 23, no. 2, pp. 129-139,2002.

[4] H. Wang, S. Li, and Y. Wang," Generalized quotient image", IEEE. Comput. Vis. Pattern Recognit., vol. 2, pp. 498-505,2004.

[5] M. Savvides, B. Kumar, and P. Khosla," Corefaces Robust shift invariant PCA based correlation filter for illumination tolerant face recognition", IEEE, Comput. Vis. Pattern Recognit., vol. 2, pp. 834-841, 2004.

[6] Anil Jain, Karthik Nandakumar , Arun Ross," Score normalization in multimodal biometric systems", Science Direct,Pattern Recognition 38,2270 - 2285,2005.

[7] C. Xie, M. Savvides, and B. V. Kumar," Kernel Correlation Filter Based Redundant Class-Dependence Feature Analysis (KCFA) on FRGC2.0 Data", SpringerVerlag Berlin Heidelberg, AMFG 2005, LNCS 3723, pp. 32-43, 2005.

[8] Xiaoyang Tan and Bill Triggs," Fusing gabor and LBP feature set for kernel-based face recognition", IEEE Int.Workshop Anal. Model. Face Gestures, pp. 235-249, 2007.

[9] Karthik Nandakumar," Likelihood Ratio Based Biometric Score Fusion", To appear in IEEE Transactions on Pattern Analysis and Machine Intelligence, 2007.

[10] Jian Yang, Chengjun Liu," Color image discriminant models and algorithms for face recognition", IEEE Trans. Neural Netw., vol. 19, no. 12, pp.20882098,2008.

[11] Zhiming Liu, Chengjun Liu," Robust face recognition using color information", Advance Biometric, vol. 5558/2009, pp. 122-131,2009.

[12] Yu Su, Shiguang Shan, X. Chen, and W. Gao," Hierarchical ensemble of global and local classifiers for face recognition", IEEE Trans. Image Process., vol. 18, no. 8, pp. 1885-1896,2009.

[13] Wonjun Hwang, Haitao Wang, Hyunwoo Kim," Face Recognition System Using Multiple Face Model of Hybrid Fourier Feature Under Uncontrolled Illumination Variation", IEEE Transactions On Image Processing, Vol. 20, No. 4,2011

[14] M. M. Mohie El-Din1, M.Y. El Nahas2 and H. A. El Shenbary," Hybrid Framework for Robust Multimodal Face Recognition", IJCSI International Journal of Computer Science Issues, Vol. 10, Issue 2, No 2,2013.

[15] Radoslav Vargic," DISCRETE Hybrid wavelet TRANSFORMS and their use for image compression", Department of telecommunications, FEI STU, Ilkovičova 3, 81219 Bratislava, Slovakia,2013.

[16] S.Anila ,Dr.N.Devarajan,” Preprocessing Technique for Face Recognition Applications under Varying Illumination Conditions", Global Journal of Computer Science and Technology Graphics \& Vision ,Volume 12 Issue 11 Version 1.0 Year 2012.

[17] R.J.E. Merry," Wavelet Theory and Applications A literature study", Eindhoven University of Technology Department of Mechanical Engineering Control Systems Technology Group Eindhoven, June 7, 2005.

[18] H. B. kekre and Kavita Sonawane," Retrieval of Images Using DCT and DCT Wavelet Over Image Blocks", International Journal of Advanced Computer Science and Applications, Vol. 2, No. 10, 2011.

[19] F. S. Samaria and A. C. Harter, "Parameterisation of a stochastic model for human face identification", Proc. of $2^{\text {nd }}$ IEEE workshop on Applications of Computer Vision, pp. 138-142, 1994.

[20] Derzu Omaia , JanKees v. d. Poel, Leonardo V. Batista, "2D-DCT Distance Based Face Recognition Using a Reduced Number of Coefficients", PPGI / DI / UFPB PPGEM / DEM / UFPB Brasil,2008. 